\title{
Utility of plasma Neurofilament light as a diagnostic and prognostic biomarker of the postural instability gait disorder motor subtype in early Parkinson's disease
}

Adeline Su Lyn $\mathrm{Ng}^{1,2^{*+}}$, Yi Jayne Tan ${ }^{1 \dagger}$, Alisa Cui Wen Yong ${ }^{1}$, Seyed Ehsan Saffari ${ }^{3}$, Zhonghao Lu', Ebonne Yulin $\mathrm{Ng}^{4}$, Samuel Yong Ern Ng${ }^{1}$, Nicole Shuang Yu Chia', Xinyi Choi ${ }^{4}$, Dede Heng ${ }^{4}$, Shermyn Neo', Zheyu Xu', Nicole Chwee Har Keong ${ }^{2,5}$, Kay Yaw Tay ${ }^{1}$, Wing Lok Au', Louis Chew Seng Tan ${ }^{1 \dagger}$ and Eng-King Tan ${ }^{2,4 \dagger}$

\begin{abstract}
Background: The main motor subtypes of Parkinson's disease (PD) include tremor-dominant (TD) and postural instability gait disorder (PIGD), with varying disease course that warrant the development of biomarkers capable of predicting progression according to motor subtype. The PIGD subtype is associated with a poorer prognosis, hence identification of a biomarker associated with PIGD is clinically relevant. Neurofilament light (NfL) chain is a potential biomarker of disease severity in neurological disorders including PD. However, no study has investigated NfL and PD motor subtypes. Here, we aimed to investigate the diagnostic and prognostic utility of plasma NfL for PD motor subtypes in early Parkinson's disease. Given the higher risk for cognitive and motor decline in PIGD, we hypothesized that plasma NfL is a potential biomarker for PIGD.

Methods: Plasma NfL was measured in 199 participants (149 PD and 50 healthy controls, HC) using an ultrasensitive single molecule array. Patients were classified into TD or PIGD based on MDS-UPDRS components. After 2 years, 115 patients were reassessed. Association between NfL and clinical measures in PIGD and TD at baseline and at 2-year follow-up were analysed.

Results: At baseline, plasma NfL levels were higher in PD than HC ( $8.8 \pm 3.4 \mathrm{vs} 16.2 \pm 7.6 \mathrm{pg} / \mathrm{ml}, p<0.0001)$, and differentiated PD from HC with a good diagnostic accuracy (AUC $=0.833, p<0.001$ ). At 2 years, NfL was higher in PIGD than TD (18.4 \pm 14.5 vs $12.6 \pm 4.4 \mathrm{pg} / \mathrm{ml}, p=0.039)$. Within the PIGD group, higher NfL associated significantly with worse global cognition and UPDRS motor scores at baseline, and was able to predict motor and cognitive decline at a mean follow-up duration of 1.9 years, controlled for age, sex and disease duration.

(Continued on next page)
\end{abstract}

\footnotetext{
* Correspondence: adeline.ng.s.l@singhealth.com.sg

${ }^{\dagger}$ Adeline Su Lyn Ng and Yi Jayne Tan are joint first authors

${ }^{+}$Louis Chew Seng Tan and Eng-King Tan are joint senior authors

'Department of Neurology, National Neuroscience Institute, Tan Tock Seng

Hospital, 11 Jalan Tan Tock Seng, Bukit Merah 308433, Singapore

${ }^{2}$ Neuroscience and Behavioural Disorders Program, Duke-NUS Medical

School, 8 College Road, Bukit Merah 169857, Singapore

Full list of author information is available at the end of the article
}

C C The Author(s). 2020 Open Access This article is licensed under a Creative Commons Attribution 4.0 International License, which permits use, sharing, adaptation, distribution and reproduction in any medium or format, as long as you give appropriate credit to the original author(s) and the source, provide a link to the Creative Commons licence, and indicate if changes were made. The images or other third party material in this article are included in the article's Creative Commons licence, unless indicated otherwise in a credit line to the material. If material is not included in the article's Creative Commons licence and your intended use is not permitted by statutory regulation or exceeds the permitted use, you will need to obtain permission directly from the copyright holder. To view a copy of this licence, visit http://creativecommons.org/licenses/by/4.0/ The Creative Commons Public Domain Dedication waiver (http://creativecommons.org/publicdomain/zero/1.0/) applies to the data made available in this article, unless otherwise stated in a credit line to the data. 
(Continued from previous page)

Conclusions: In this longitudinal study, we demonstrated for the first time the potential utility of plasma NfL as a diagnostic and prognostic biomarker in PIGD even at early stages of PD. These important novel findings will require further confirmation in larger, longitudinal PD cohorts.

Keywords: Parkinson's disease, Motor subtype, PIGD, Biomarkers, Neurofilament light chain, Cognition

\section{Background}

Neuroaxonal loss remains the pathological substrate of permanent disability in neurological disorders. Reliable quantification of axonal loss is important for both disease monitoring and prognostication. The neurofilament proteins play an important role as their levels rise with neuroaxonal damage, indicating neuroaxonal injury independent of causal pathways [1]. Out of the neurofilament proteins, neurofilament light (NfL) chain has been recognized as a possible marker of axonal injury in various neurological disorders [1], including multiple sclerosis [2], motor neurone disease [3, 4], frontotemporal dementia spectrum disorders $[5,6]$, traumatic brain injury [7], and cerebrovascular disease [8]. In Parkinson's disease (PD), blood NfL has been reported to be higher in cases vs controls $[9,10]$, and shown to be able to distinguish PD from atypical PD (APD) [11, 12]. NfL has also been found to associate with longer disease duration, more aggravated motor symptoms [11], and correlate with motor and/or cognitive decline [13] in PD.

Classification of PD motor subtypes based on the rate of clinical progression allows for both closer monitoring and earlier implementation of appropriate management for the poorer prognostic motor subtypes. The postural instability and gait disorder (PIGD) subtype has been associated with greater clinical progression and cognitive dysfunction, whereas tremor dominant (TD) PD is associated with a better prognosis $[14,15]$. The heterogenous clinical phenotypes and varying disease course seen in PD patients highlights the need for biomarkers that can predict disease progression according to motor subtypes more accurately. Given the greater risk for cognitive impairment and motor progression in PIGD [14-16], we hypothesized that NfL may be a potential biomarker for the PIGD subtype. Furthermore, we aimed to determine if this association with PD motor subtypes can also be observed in early stages of the disease, which has important clinical implications for patient management and prognostication. To address these gaps, we conducted the first longitudinal study to investigate the association of NfL levels with cognitive and motor outcomes with PIGD and compared this with tremor-dominant PD.

\section{Methods}

\section{Clinical recruitment}

Participants were recruited from the National Neuroscience Institute, Singapore, between November 2014 and
July 2018. All PD patients fulfilled the National Institute of Neurological Disorders and Stroke (NINDS) criteria for the diagnosis of PD. [17] The Early Parkinson's Disease Longitudinal Singapore (PALS) study is an ongoing prospective cohort study analysing the progression of early PD over a follow-up period of 5 years. Patients were defined as 'early PD' using the following inclusion criteria: (i) motor symptoms $\leq 2$ years, and (ii) carrying a diagnosis of PD of $\leq 1$ year using the NINDS criteria as diagnosed by a movement disorders specialist. Healthy controls (HC) were recruited from the community and were free of significant neurological, psychiatric or systemic disease. Ethics approval was obtained from the Singapore Health Services Centralised Institutional Review Board (CIRB) for the use of human participants in this study, and all participants provided informed written consent.

\section{Clinical measures at baseline and follow-up}

The Movement Disorders Society Unified Parkinson's Disease Rating Scale (MDS-UPDRS) [18] was conducted by trained movement disorder specialists while participants were in the "ON" state, and scores were obtained thrice yearly. Functional status was determined using the Hoehn and Yahr (H\&Y) rating scale [19] and motor severity using the MDS-UPDRS Part III motor components [18]. Patients were classified into motor subtypes of "tremor dominant" (TD), "postural instability and gait disorders" (PIGD) or "indeterminate" based on MDS-UPDRS part II and III components [20]. Patients classified as "indeterminate" were excluded from analysis. Global cognition was measured using the Mini-Mental State Examination (MMSE) [21] and Montreal Cognitive Assessment (MoCA) [22] tools.

\section{Plasma NfL measurement}

Blood samples were collected at baseline and annually. A total of 199 participants (149 PD and $50 \mathrm{HC}$ ) had their plasma NfL levels measured at baseline. Seventy-six PD patients (30 TD and 46 PIGD) had plasma NfL measured at the 2-year mark. EDTA blood was centrifuged at $1500 \mathrm{~g}$ for $15 \mathrm{~min}$ within $1 \mathrm{~h}$ after collection. Plasma was aliquoted and stored at $-80^{\circ} \mathrm{C}$ until further analysis. Samples were thawed only once for NfL measurement. NfL levels were measured using ultrasensitive single molecule array (Simoa) Human NfL assay and Simoa HD-1 Analyzer (Quanterix, MA), according to the manufacturer's protocol. Coefficient of variation of samples measured in duplicates was $<10 \%$. 
Two quality-control samples with high and low NfL concentrations, provided in the kit, were measured in each run and were in the expected range.

\section{Statistical analysis}

Demographics and clinical characteristics were compared between PD and $\mathrm{HC}$ using independent two-sample t-test and Chi-square test for continuous and categorical variables, respectively. Correlations between clinical data and NfL levels were assessed using Spearman's rank order correlation. The three motor subtypes (TD vs. PIGD vs. Indeterminate) were compared for similar parameters by analysis of variance (ANOVA) and Chi-square test for continuous and categorical variables, respectively. Natural logarithm (Ln) transformation was performed to reduce right skewness for plasma NfL levels for subsequent analysis. Multivariable linear regression analysis was performed to investigate the association between NfL levels (and its interaction with motor-subtype) and clinical outcomes, controlling for age, sex and disease duration as potential confounders. The diagnostic accuracy of plasma NfL (as a predictive marker of PD vs controls) was assessed via receiver operating characteristic (ROC) analysis. Multivariable logistic regression analysis was conducted to investigate the association between standardized Ln-NfL level (and its interaction with age) and clinical diagnosis (PD vs controls), and contour plot was generated based on the calculated predicted probabilities. Associations between baseline plasma NfL levels (and its interaction with motor subtypes) and change in motor and cognitive outcomes over time were investigated by linear mixed models, adjusted for age, sex and disease duration. Auto Regressive (AR-1) variance-covariance structure method was used to account for repeated measures outcomes. Statistical significance was set at $p<0.05$, and all tests were two-sided. Statistical analysis was performed in SAS version 9.4 for Windows (SAS Institute Inc., Cary, NC, USA).

\section{Results}

\section{Baseline characteristics}

Participant demographics and clinical features at baseline are shown in Table 1 . Plasma NfL positively correlated with age in PD ( $\mathrm{rs}=0.557, p<0.001)$ and $\mathrm{HC}(\mathrm{rs}=$ 0.294, $p=0.038$ ), but not sex in both PD and HC. There was a negative association between NfL and disease duration ( $\mathrm{rs}=-0.291, p<0.001$ ) but not levodopa equivalent daily dosage (LEDD) ( $\mathrm{rs}=0.036, p=0.676)$ in PD.

\section{Analyses between PD patients and HC}

Baseline plasma NfL levels were significantly elevated in PD compared to $\mathrm{HC}$ in univariate $(p<0.001$; Table 1$)$ and multivariable analyses (adjusted for age and sex, $p<$ 0.001; Fig. 1a). ROC analysis showed that plasma NfL could discriminate PD from $\mathrm{HC}$ with area under the curve (AUC): 0.833, 95\% CI: 0.774-0.893, sensitivity = $60 \%$, specificity $=90 \%$; Fig. 2 ).

\section{Analyses between PIGD and TD PD}

While baseline plasma NfL levels did not differ significantly between TD and PIGD motor subtypes in univariate (Table 1) and multivariable analyses (adjusted for age, sex and disease duration, $p=0.687$; Fig. 1b), we found that NfL levels were significantly higher in the

Table 1 Demographic and clinical characteristics of all subjects at baseline

\begin{tabular}{|c|c|c|c|c|c|}
\hline Parameter & $\mathrm{HC}$ & PD & TD & PIGD & Indeterminate \\
\hline Subjects, $n$ & 50 & 149 & 70 & 55 & 24 \\
\hline Age, years & $57.2 \pm 5.4^{a}$ & $64.0 \pm 8.3^{\mathrm{b}^{* *}}$ & $64.0 \pm 8.0$ & $63.6 \pm 8.3$ & $65.0 \pm 9.4$ \\
\hline Male sex, n(\%) & $25(50)$ & $85(57)$ & $43(61)$ & $28(51)$ & $14(58)$ \\
\hline Age at onset, years & - & $62.9 \pm 8.5$ & $63.1 \pm 8.2$ & $62.3 \pm 8.5$ & $63.8 \pm 9.4$ \\
\hline Disease duration, years & - & $1.1 \pm 0.9$ & $0.9 \pm 0.7$ & $1.3 \pm 1.3^{\mathrm{d}^{*}}$ & $1.2 \pm 0.6^{c^{*}}$ \\
\hline LEDD & - & $176.5 \pm 135.3$ & $172.6 \pm 133.3$ & $182.9 \pm 142.4$ & $172.3 \pm 129.2$ \\
\hline MDS-UPDRS Part II: ADLs & - & $4.1 \pm 3.2$ & $3.7 \pm 2.9$ & $4.7 \pm 3.5$ & $4.0 \pm 3.2$ \\
\hline MDS-UPDRS Part III: Motor & - & $21.4 \pm 9.3$ & $21.1 \pm 10.0$ & $22.0 \pm 9.5$ & $20.7 \pm 7.0$ \\
\hline H\&Y stage, & - & $1.8 \pm 0.4$ & $1.7 \pm 0.4$ & $1.9 \pm 0.4^{\mathrm{d}^{*}}$ & $1.9 \pm 0.4^{c^{*}}$ \\
\hline MMSE & $29.0 \pm 1.3$ & $26.8 \pm 2.8^{b^{* *}}$ & $27.0 \pm 2.5$ & $26.6 \pm 3.4$ & $27.0 \pm 2.0$ \\
\hline MoCA & $27.5 \pm 2.0$ & $25.1 \pm 3.5^{b^{* *}}$ & $25.3 \pm 3.3$ & $24.5 \pm 3.6$ & $25.6 \pm 4.2$ \\
\hline Plasma NfL, pg/ml & $8.8 \pm 3.4$ & $16.2 \pm 7.6^{\mathrm{b}^{* *}}$ & $15.8 \pm 7.0$ & $15.8 \pm 7.1$ & $18.2 \pm 10.3$ \\
\hline
\end{tabular}

Abbreviations: PD Parkinson's Disease; HC Healthy Control; TD Tremor-Dominant; PIGD Postural Instability Gait Disorder; LEDD Levodopa Equivalent Dose; MDSUPDRS Movement Disorder Society Unified Parkinson's Disease Rating Scale; ADL Activities of Daily Living; H\&Y stage Hoehn and Yahr stage; MMSE Mini Menta State Examination; MoCA Montreal Cognitive Assessment; NfL Neurofilament light chain protein. ${ }^{\text {a }}$ Continuous variables reported as mean \pm standard deviation; Categorical variables reported as $\mathrm{n}(\%)$

$* P<0.05, * * P<0.01$ using Analysis of variance for continuous variables; Chi-squared test for categorical variable for comparisons of variables between ${ }^{\text {a }}$ PD and HC, ${ }^{c}$ TD, PIGD and Indeterminate subtypes, ${ }^{d}$ TD and PIGD subtypes 

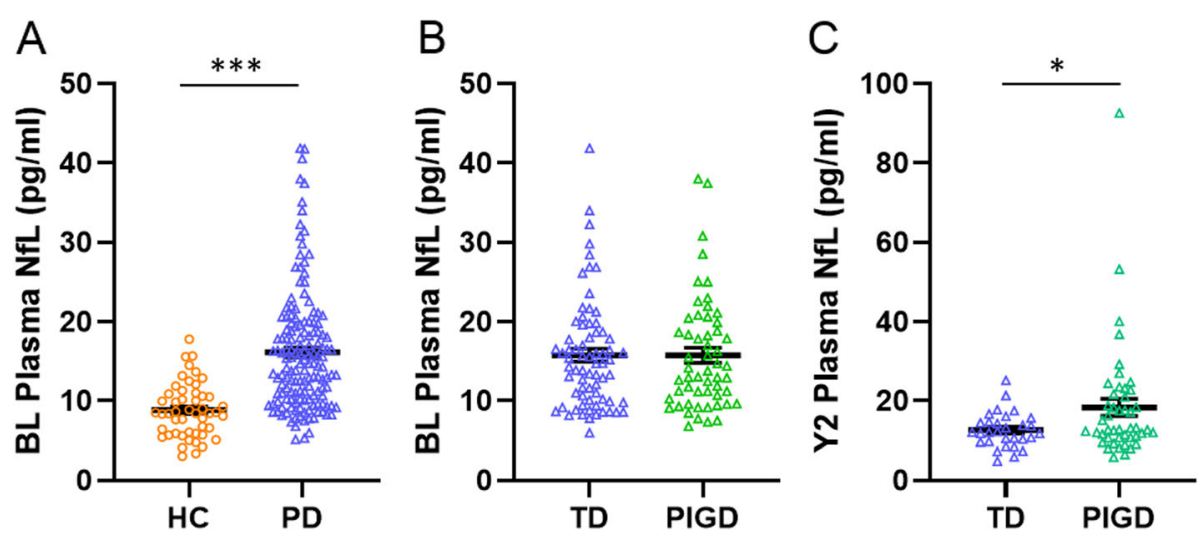

Fig. 1 Plasma NfL in healthy controls and patients with Parkinson's disease. a At baseline, plasma NfL was significantly increased in PD compared to HC, adjusting for age and sex; $\mathbf{b}$ but not between TD and PIGD, adjusting for age, sex and disease duration. $\mathbf{c}$ At year 2, plasma NfL was significantly increased in PIGD compared to TD, adjusting for age, sex and disease duration. Abbreviations: BL, Baseline; PD, Parkinson's Disease; HC, Healthy Control; NfL, Neurofilament light chain protein; TD, Tremor-Dominant; PIGD, Postural Instability Gait Disorder; Y2, Year 2

PIGD than TD group at Year $2(18.4 \pm 14.5$ vs $12.6 \pm 4.4$ $\mathrm{pg} / \mathrm{ml}, p=0.025$, adjusted for age, sex and disease duration; Fig. 1cc). ROC analysis showed that plasma NfL could discriminate between the two motor subtypes with an AUC of $0.656,95 \%$ CI: $0.533-0.779$, sensitivity $=90 \%$, specificity $=30.4 \%$. Across all PD patients, NfL did not show significant association with cognitive or motor measures at baseline. In the PIGD group however, higher NfL levels significantly associated with worse MoCA and UPDRS Part III motor scores (Table 2), controlling for age, sex and disease duration. These results remained after adding LEDD as a covariate.

\section{Two-year longitudinal outcomes between NfL levels and PD motor subtypes}

Out of 149 PD patients, 115 patients (53 TD and 45 PIGD) with available follow-up data at 2 years were included in subsequent analysis to assess the prognostic utility of plasma NfL. The mean duration of follow-up was 1.9 years. Longitudinal data of plasma NfL, cognitive

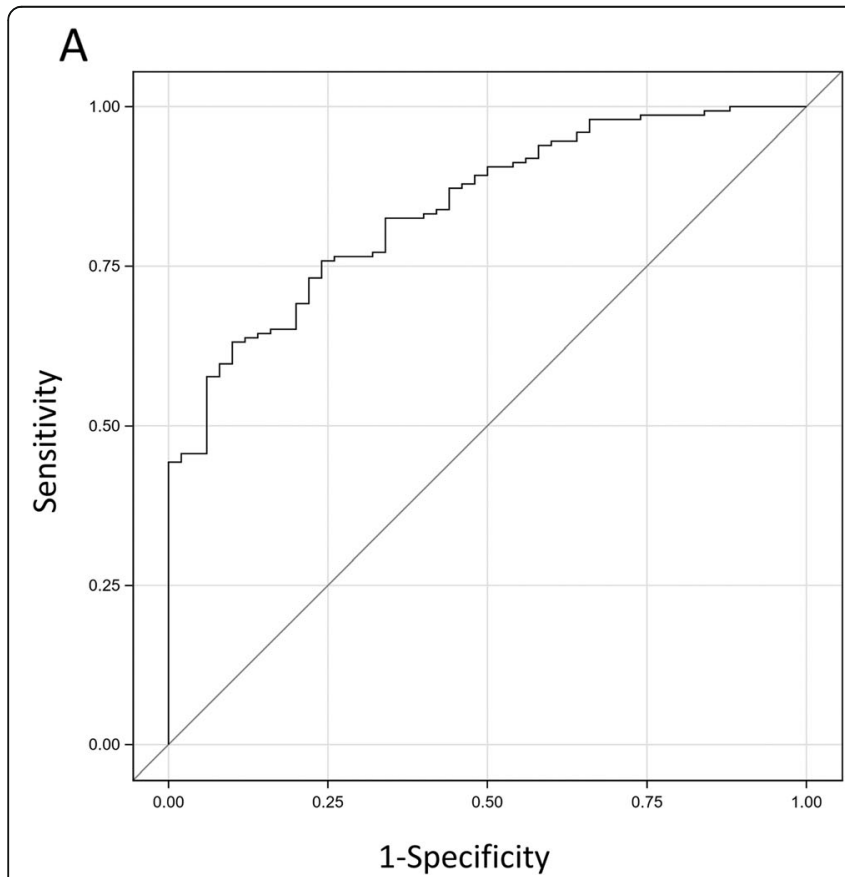

B

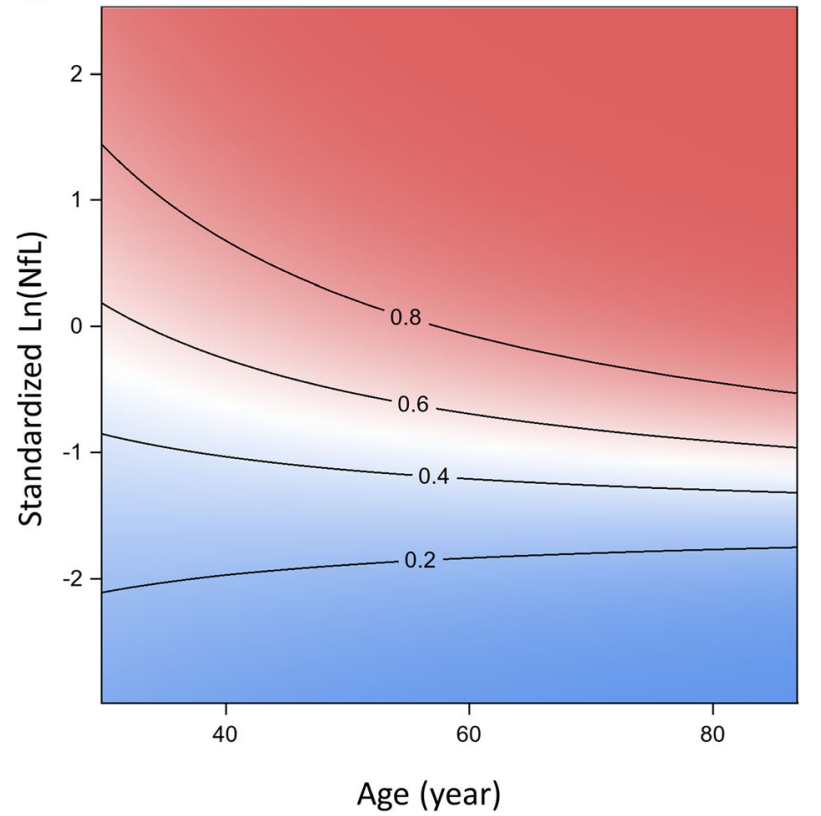

Fig. 2 Baseline plasma NfL discriminates PD from healthy controls. a Receiver operating characteristic curve analysis in PD vs HC b Contour plot showing predicted probability of clinical diagnosis of PD at different NfL levels and age group. The darker shade of red indicates higher risk and darker blue indicates lower risk of PD. The solid line depicts the risk of PD using multivariable logistic regression model adjusted NfL $x$ age interaction term. Abbreviations: PD, Parkinson's Disease; HC, Healthy Control; NfL, Neurofilament light chain protein 
Table 2 Multivariable analysis on baseline plasma NfL levels with cognitive and motor outcomes in PD patients

\begin{tabular}{llll}
\hline Group & MDS-UPDRS Part III (Motor) & MoCA \\
& $\beta^{\mathrm{b}}(95 \% \mathrm{Cl})$ & $\mathrm{H} \& \mathrm{Y}$ & $\beta(95 \% \mathrm{Cl})$ \\
\hline Total PD & $-0.567(-4.775,3.642)$ & $-0.029(-0.214,0.157)$ & $-0.455(-1.995,1.086)$ \\
TD & $-6.815(-14.036,0.407)$ & $-0.188(-0.498,0.121)$ & $1.437(-0.757,3.631)$ \\
PIGD & $10.687^{* *}(3.325,18.050)$ & $0.292(-0.021,0.605)$ & $-3.168^{*}(-5.954,-0.382)$
\end{tabular}

Abbreviations: PD Parkinson's Disease; TD Tremor-Dominant; PIGD Postural Instability Gait Disorder; H\&Y stage Hoehn and Yahr stage; MDS-UPDRS Movement Disorder Society Unified Parkinson's Disease Rating Scale; MoCA Montreal Cognitive Assessment; Cl Confidence Interval; NfL, Neurofilament light chain protein

${ }^{a}$ MDS-UPDRS Part III (Motor) scores, H\&Y stages and MoCA scores of PD patients are the outcome variables

${ }^{b}$ Beta coefficient (and $95 \% \mathrm{Cl}$ ) of Ln-NfL level; Multivariable linear regression adjusted for age, sex and disease duration

* $P<0.05$, ** $P<0.01$

and motor scores are summarised in Table 3. Using linear mixed models, we tested the association between interaction of baseline plasma NfL levels with TD and PIGD subtypes and clinical outcomes in total PD over time, adjusting for baseline characteristics such as age, sex and disease duration. Across all PD patients, baseline plasma NfL did not associate significantly with motor and cognition outcome over time. However, higher baseline plasma NfL levels significantly associated with worse motor and cognition outcomes over time in the PIGD group (Table 4), but not in the TD group. In the PIGD group, every one unit increase in Ln-NfL $(=2.72$ increase in plasma $\mathrm{NfL}$ level) resulted in a corresponding 9.73 unit increase in motor outcome (as assessed by MDSUPDRS part III) and 2.09 unit decrease in cognitive outcome (as assessed by MMSE), controlling for baseline age, sex and disease duration.

Table 3 Plasma NfL, cognitive and motor data in PD patients over different timepoints

\begin{tabular}{llllll}
\hline Group & Values & Time & & \multirow{2}{l}{$\begin{array}{l}\text { Trend } \\
p \text { value }\end{array}$} \\
\cline { 3 - 5 } & & Baseline & Year 1 & Year 2 & \\
\hline Total PD & NfL & $16.16 \pm 0.62$ & - & $16.11 \pm 1.37$ & 0.2881 \\
& MMSE & $26.82 \pm 0.23$ & $26.58 \pm 0.25$ & $26.9 \pm 0.25$ & 0.4523 \\
& MoCA & $25.06 \pm 0.29$ & $25.05 \pm 0.31$ & $25.17 \pm 0.30$ & 0.9324 \\
& Motor & $21.32 \pm 0.76$ & $24.76 \pm 0.84$ & $25.96 \pm 1.03$ & $<0.001$ \\
TD & NfL & $15.79 \pm 0.83$ & - & $12.62 \pm 0.81$ & 0.1865 \\
& MMSE & $26.99 \pm 0.30$ & $26.88 \pm 0.28$ & $27.13 \pm 0.29$ & 0.9128 \\
& MoCA & $25.32 \pm 0.39$ & $25.35 \pm 0.39$ & $25.42 \pm 0.38$ & 0.8401 \\
PIGD & Motor & $20.99 \pm 1.19$ & $24.40 \pm 1.20$ & $25.46 \pm 1.37$ & $<0.001$ \\
& NfL & $15.76 \pm 0.96$ & - & $18.39 \pm 2.15$ & 0.0474 \\
& MMSE & $26.55 \pm 0.46$ & $26.06 \pm 0.54$ & $26.5 \pm 0.46$ & 0.4865 \\
& MoCA & $24.47 \pm 0.48$ & $24.38 \pm 0.63$ & $24.61 \pm 0.54$ & 0.8705 \\
& Motor & $22.02 \pm 1.28$ & $26.17 \pm 1.44$ & $26.80 \pm 1.77$ & $<0.001$ \\
\hline
\end{tabular}

Abbreviations: PD Parkinson's Disease; TD Tremor-Dominant; PIGD Postural Instability Gait Disorder; Motor Movement Disorder Society Unified Parkinson's Disease Rating Scale (MDS-UPDRS) part III scores; MoCA Montreal Cognitive Assessment; NfL Neurofilament light chain protein

Values represent the mean $\pm S D$

\section{Discussion}

The PIGD motor subtype of PD is associated with a poorer prognosis and greater clinical progression in PD. In this 2-year longitudinal study, we investigated if plasma NfL may be a potential biomarker for the PIGD subtype of PD in early stages of disease. We demonstrated for the first time that higher NfL levels at baseline significantly associate with and predict greater global cognitive and motor impairment in patients with PIGD both at baseline and over 2 years, accounting for age, sex and disease duration. Furthermore, we were able to provide quantification of the extent to which baseline NfL can predict cognitive or motor decline within a relatively short follow-up period of 2 years.

While limited studies have previously reported higher blood NfL levels in PD compared to controls, our study has included a comparable number, if not substantially more participants than these [10-12]. Importantly, we were able to show that plasma NfL can discriminate PD from $\mathrm{HC}$ with high diagnostic accuracy even at early stages of the disease. NfL levels were measured in our patients at baseline, all of whom had a mean disease duration of 1.1 years at time of study recruitment, compared to other studies investigating NfL in PD which had mean disease duration ranging from 2 to 3 years [12], to 7 to 9 years [11, 13].

Additionally, NfL has previously shown only modest or inconsistent correlation with UPDRS Part III motor scores in PD [11, 13], possibly related to the fact that the PD groups in these studies were not analysed according to motor subtype. Higher NfL levels in PD can be explained given that NfL remains a well-established biomarker of neuroaxonal loss [1], and profound dopaminergic axonal loss has been shown to occur even at early stages of PD. [23] In vitro, induced pluripotent stem cell (iPSC)-derived dopaminergic neurons from patients carrying alphasynuclein $(S N C A)$ triplications or leucine-rich repeat kinase (LRRK2) G2019S mutations have also demonstrated evidence of neuroaxonal degeneration [24].

While plasma NfL levels were not different between TD and PIGD at baseline, this was not surprising given that both PD subtypes may appear similar at very early 
Table 4 Multivariable analysis on baseline plasma NfL levels with cognitive and motor outcomes in PD patients over time

\begin{tabular}{|c|c|c|c|c|}
\hline \multirow[t]{2}{*}{ Outcomes } & \multicolumn{2}{|c|}{ MDS-UPDRS Part III (Motor) ${ }^{a}$} & \multicolumn{2}{|l|}{ MMSE } \\
\hline & $\overline{\beta(95 \% \mathrm{Cl})^{\mathrm{b}}}$ & $\overline{p \text { value }}$ & $\overline{\beta(95 \% \mathrm{Cl})}$ & $\overline{p \text { value }}$ \\
\hline $\mathrm{NfL}(\mathrm{PIGD})$ & $9.73(3.65,15.81)$ & 0.002 & $-2.09(-3.90,-0.28)$ & 0.024 \\
\hline NfL (TD) & $-3.90(-9.36,1.57)$ & 0.161 & $0.50(-1.14,2.13)$ & 0.549 \\
\hline NfL $\times$ Motor Subtype & $13.6(6.16,21.1)$ & $<0.001$ & $-2.59(-4.81,-0.36)$ & 0.023 \\
\hline Age & $0.10(-0.12,0.32)$ & 0.385 & $-0.08(-0.14,-0.01)$ & 0.025 \\
\hline Gender & $-2.75(-5.84,0.35)$ & 0.081 & $-0.09(-1.01,0.83)$ & 0.839 \\
\hline Disease duration & $-0.75(-2.43,0.93)$ & 0.379 & $0.17(-0.34,0.69)$ & 0.506 \\
\hline
\end{tabular}

Abbreviations: PD Parkinson's Disease; TD Tremor-Dominant; PIGD Postural Instability Gait Disorder; MDS-UPDRS Movement Disorder Society Unified Parkinson's Disease Rating Scale; MMSE Mini Mental State Examination; Cl Confidence Interval; NfL Neurofilament light chain protein

${ }^{\text {a }}$ MDS-UPDRS Part III (Motor) and MMSE scores of PD patients over 2 years are the outcome variables

${ }^{\mathrm{b}}$ Beta coefficient (and 95\% Cl) of Ln-NfL level; Linear mixed models adjusted for age, sex, disease duration and NfL x motor subtype interaction

disease stages. In 2 years, a significant difference between NfL levels in both groups appeared, suggesting that PIGD may have progressed to a greater extent pathophysiologically than the more 'benign' TD subtype. Currently, there are no molecular biomarkers available that can reliably discriminate PD motor subtypes without reliance on clinical presentation, which often fluctuates and necessitate more objective biomarker-based discrimination. Multiple studies have investigated cerebrospinal fluid (CSF) biomarkers in PD motor subtypes; CSF A $\beta$ 42 and P-tau181 concentrations have associated with the PIGD but not with TD or intermediate phenotypes [25], while CSF $\alpha$-synuclein levels were found to be significantly lower in non-TD or PIGD phenotypes compared with the TD phenotype [26, 27]. Fewer studies, however, have attempted to identify blood-based biomarkers that can similarly discriminate between PD motor subtypes, a significant gap given the less invasive nature of blood sampling vs CSF sampling. Patients with the PIGD subtype were reported to have lower plasma $A \beta-42$ and higher plasma $\alpha$-synuclein levels than the TD group [28], while our group has previously reported higher serum uric acid levels in the TD motor subtype [29]. Our results here suggest that approximately 3 years after symptom onset, plasma NfL may discriminate TD and PIGD subtypes, and potentially act as a diagnostic biomarker for PIGD, which will be useful particularly in the clinical trial setting.

Our findings are consistent with the known clinical hallmarks of PIGD, which is characterized by more severe disease manifestations at diagnosis, greater risk of cognitive impairment and neuropsychiatric symptoms than TD [14-16], and more rapid disease progression than the less malignant clinical subtype [30]. Importantly, clinical severity of PIGD has been proposed as a useful indicator of severity and prognosis in PD by itself [14], suggesting the more crucial need for a biomarker of disease intensity in PIGD, rather than in TD.

While we do not have postmortem confirmation of PD in the study, all our PIGD patients satisfied the clinical criteria for PD and were responsive to levodopa with no clinical features to suggest a Parkinson-Plus syndrome. At 2-year follow-up, clinical diagnosis of PIGD remained in this group of patients. Additionally, we accounted for LEDD in our analyses. Despite the moderate sensitivity of NfL in discriminating PD from $\mathrm{HC}$, its high specificity remains clinically useful for initial screening of $\mathrm{PD}$ and PD subtypes from controls, together with other supportive clinical features with high sensitivity such as anosmia. Hence, NfL can be included in the initial workup of patients when diagnosis remains uncertain. The Early Parkinson's Disease Longitudinal Singapore (PALS) study is an ongoing prospective cohort study analysing the progression of early PD over a follow-up period of 5 years. This longitudinal study will allow us to systematically analyse the performance of plasma NfL in combination with other biomarkers reflecting different pathophysiological processes in PD (e.g. plasma tau, alpha-synuclein and uric acid etc), which may improve its discriminatory performance. Additionally, blood samples collected at future timepoints will be analysed in the same sitting as far as possible to reduce batch-tobatch variability, with inter-assay control included in future runs.

\section{Conclusions}

Here, we highlight a novel relationship between plasma $\mathrm{NfL}$ and PD motor subtypes by demonstrating an association between NfL and cognitive and motor measures over 2 years in PIGD. Overall, our results suggest that plasma NfL can be a potential biomarker to facilitate closer monitoring and management for PIGD, a poorer prognostic motor subtype of PD. The heterogeneity of PD clinical phenotypes with varying disease trajectories highlights the need for better biomarkers to facilitate more accurate monitoring and prognostication of PD subtypes, relevant in our push towards personalized medicine. These important findings offer great opportunities for further confirmation in larger, longitudinal PD cohorts. 


\section{Abbreviations}

APD: Atypical PD; CSF: Cerebrospinal fluid; HC: Healthy controls; H\&Y stage: Hoehn and Yahr stage; LEDD: Levodopa equivalent dose; MDSUPDRS: Movement disorder society unified parkinson's disease rating scale; MMSE: Mini mental state examination; MoCA: Montreal cognitive assessment; NINDS: National institute of neurological disorders and stroke;

NfL: Neurofilament light chain protein; PALS: Parkinson's disease longitudinal singapore; PD: Parkinson's disease; PIGD: Postural instability gait disorder; ROC: Receiver operating characteristic; SIMOA: Single molecule array; SNCA: Alpha-synuclein; LRRK2: Leucine-rich repeat kinase; TD: Tremordominant

\section{Acknowledgements}

The authors thank our patients and their families for their valuable contribution to the study.

\section{Authors' contributions}

ASLN and YJT conceived and designed the study. YJT performed the experiments. SN, ZYX, NCHK, KYT, WLA, EKT and LCST contributed in participant examination and recruitment. ACWY, ZHL, SYEN, NSYC, XYC, DDH, EYLN and YJT contributed in data collection. YJT, SES and ASLN analysed and interpreted the data. ASLN and YJT drafted the manuscript. EKT and LCST revised the manuscript. All authors read and approved the final manuscript.

\section{Funding}

This study was funded by Singapore's National Medical Research Council (ASLN by the Clinician-Scientist New Investigator Grant (CNIG/1165/2017) and Transition Award (MOH-TA18may-0003); EKT and LCST by the Singapore Translational Research (STaR) Investigator award (NMRC/STaR/014/2013), and Parkinson's disease Translational and Clinical Research flagship grant (TCR12dec010)).

\section{Availability of data and materials}

The datasets used and/or analysed in the current study are available from the corresponding author on reasonable request

\section{Ethics approval and consent to participate}

Ethics approval was obtained from the Singapore Health Services Centralised Institutional Review Board (CIRB) for the use of human participants in this study, and all participants provided informed written consent.

\section{Consent for publication}

All authors have approved of the manuscript and agree with its submission.

\section{Competing interests}

The authors declare no competing interests.

\section{Author details}

'Department of Neurology, National Neuroscience Institute, Tan Tock Seng Hospital, 11 Jalan Tan Tock Seng, Bukit Merah 308433, Singapore. ${ }^{2}$ Neuroscience and Behavioural Disorders Program, Duke-NUS Medical School, 8 College Road, Bukit Merah 169857, Singapore. ${ }^{3}$ Center for Quantitative Medicine, Duke-NUS Medical School, Bukit Merah, Singapore. ${ }^{4}$ Department of Neurology, National Neuroscience Institute, Singapore General Hospital, 20 College Road, Bukit Merah 169856, Singapore. ${ }^{5}$ Department of Neurosurgery, National Neuroscience Institute, Tan Tock Seng Hospital, 11 Jalan Tan Tock Seng, Bukit Merah 308433, Singapore.

Received: 13 January 2020 Accepted: 28 May 2020

Published online: 05 June 2020

\section{References}

1. Khalil M, Teunissen CE, Otto M, et al. Neurofilaments as biomarkers in neurological disorders. Nat Rev Neurol. 2018;14:577.

2. Disanto G, Barro C, Benkert $P$, et al. Serum Neurofilament light: a biomarker of neuronal damage in multiple sclerosis. Ann Neurol. 2017; 81:857-70.

3. Poesen K, De Schaepdryver M, Stubendorff B, et al. Neurofilament markers for ALS correlate with extent of upper and lower motor neuron disease. Neurology. 2017;88:2302-9.
4. Gaiani A, Martinelli I, Bello L, et al. Diagnostic and prognostic biomarkers in amyotrophic lateral sclerosis: Neurofilament light chain levels in definite subtypes of disease. JAMA Neurol. 2017;74:525-32.

5. Rohrer JD, Woollacott IOC, Dick KM, et al. Serum neurofilament light chain protein is a measure of disease intensity in frontotemporal dementia. Neurology. 2016;87:1329-36.

6. Rojas JC, Karydas A, Bang J, et al. Plasma neurofilament light chain predicts progression in progressive supranuclear palsy. Ann Clin Transl Neurol. 2016; 3:216-25.

7. Shahim P, Zetterberg H, Tegner Y, Blennow K. Serum neurofilament light as a biomarker for mild traumatic brain injury in contact sports. Neurology. 2017:88:1788-94.

8. Gattringer T, Pinter D, Enzinger $C$, et al. Serum neurofilament light is sensitive to active cerebral small vessel disease. Neurology. 2017;89: 2108-14

9. Mollenhauer B, Dakna M, Liu T-Y, et al. Validation of serum neurofilament light chain as a biomarker of parkinson's disease progression. bioRxiv. Cold Spring Harbor Laboratory; Epub. 2019:762237. https://doi.org/10.1101/ 762237.

10. Oosterveld LP, Verberk IMW, Majbour NK, et al. CSF or serum neurofilament light added to a-Synuclein panel discriminates Parkinson's from controls. Mov Disord. 2020;35:288-95.

11. Hansson O, Janelidze S, Hall S, et al. Blood-based NfL: a biomarker for differential diagnosis of parkinsonian disorder. Neurology. 2017 Mar 7; 88(10):930-937 https://doi.org/10.1212/WNL.00000000000003680.

12. Marques TM, van Rumund $A$, Oeckl $P$, et al. Serum NFL discriminates Parkinson disease from atypical parkinsonisms. Neurology. 2019;92: e1479-86.

13. Lin C-H, Li C-H, Yang K-C, et al. Blood NfL: a biomarker for disease severity and progression in Parkinson disease. Neurology. 2019 Sep 10;93(11):e1104e1111. https://doi.org/10.1212/WNL.0000000000008088. Epub 2019 Aug 16.

14. van der Heeden JF, Marinus J, Martinez-Martin P, Rodriguez-Blazquez C, Geraedts VJ, van Hilten JJ. Postural instability and gait are associated with severity and prognosis of Parkinson disease. Neurology. 2016;86: 2243-50.

15. Aleksovski D, Miljkovic D, Bravi D, Antonini A. Disease progression in Parkinson subtypes: the PPMI dataset. Neurol Sci. 2018;39:1971-6.

16. Guo Y, Xu W, Liu F-T, et al. Modifiable risk factors for cognitive impairment in Parkinson's disease: a systematic review and meta-analysis of prospective cohort studies. Mov Disord. 2019:34:876-83.

17. Gelb DJ, Oliver E, Gilman S. Diagnostic criteria for Parkinson disease. Arch Neurol. 1999:56:33-9.

18. Movement Disorder Society Task Force on Rating Scales for Parkinson's Disease. The unified Parkinson's disease rating scale (UPDRS): status and recommendations. Mov Disord. 2003;18:738-50.

19. Hoehn MM, Yahr MD. Parkinsonism: onset, progression and mortality. Neurology. 1967;17:427-42

20. Stebbins GT, Goetz CG, Burn DJ, Jankovic J, Khoo TK, Tilley BC. How to identify tremor dominant and postural instability/gait difficulty groups with the movement disorder society unified Parkinson's disease rating scale: comparison with the unified Parkinson's disease rating scale. Mov Disord. 2013;28:668-70.

21. Folstein MF, Folstein SE, McHugh PR. "Mini-mental state". A practical method for grading the cognitive state of patients for the clinician. J Psychiatr Res. 1975;12:189-98.

22. Nasreddine ZS, Phillips NA, Bédirian V, et al. The Montreal cognitive assessment, MoCA: a brief screening tool for mild cognitive impairment. Am Geriatr Soc. 2005;53:695-9.

23. O'Keeffe GW, Sullivan AM. Evidence for dopaminergic axonal degeneration as an early pathological process in Parkinson's disease. Parkinsonism Relat Disord. 2018;56:9-15.

24. Lin L, Göke J, Cukuroglu E, Dranias MR, VanDongen AMJ, Stanton LW. Molecular features underlying Neurodegeneration identified through in vitro modeling of genetically diverse Parkinson's disease patients. Cell Rep. 2016;15:2411-26.

25. Kang $J-H$, Irwin DJ, Chen-Plotkin AS, et al. Association of cerebrospinal fluid $\beta$ amyloid 1-42, T-tau, P-tau181, and a-synuclein levels with clinical features of drug-naive patients with early Parkinson disease. JAMA Neurol. 2013;70:1277-87.

26. Kang J-H, Mollenhauer B, Coffey CS, et al. CSF biomarkers associated with disease heterogeneity in early Parkinson's disease: the 
Parkinson's progression markers initiative study. Acta Neuropathol. 2016;131:935-49.

27. Goldman JG, Andrews H, Amara A, et al. Cerebrospinal fluid, plasma, and saliva in the BioFIND study: relationships among biomarkers and Parkinson's disease features. Mov Disord. 2018;33:282-8.

28. Ding J, Zhang J, Wang $X$, et al. Relationship between the plasma levels of neurodegenerative proteins and motor subtypes of Parkinson's disease. J Neural Transm (Vienna). 2017;124:353-60.

29. Huang X, Ng SY-E, Chia NS-Y, et al. Serum uric acid level and its association with motor subtypes and non-motor symptoms in early Parkinson's disease: PALS study. Parkinsonism Relat Disord. 2018;55:50-4.

30. De Pablo-Fernández E, Lees AJ, Holton JL, Warner TT. Prognosis and Neuropathologic correlation of clinical subtypes of Parkinson disease. JAMA Neurol. 2019 Apr 1;76(4):470-79. https://doi.org/10.1001/jamaneurol.2018 4377.

\section{Publisher's Note}

Springer Nature remains neutral with regard to jurisdictional claims in published maps and institutional affiliations.

Ready to submit your research? Choose BMC and benefit from:

- fast, convenient online submission

- thorough peer review by experienced researchers in your field

- rapid publication on acceptance

- support for research data, including large and complex data types

- gold Open Access which fosters wider collaboration and increased citations

- maximum visibility for your research: over $100 \mathrm{M}$ website views per year

At BMC, research is always in progress.

Learn more biomedcentral.com/submissions 